\title{
Nonlinear viscoelastic oscillations of single- mass system at power excitation
}

\author{
Nurilla Noraliev $^{1 *}$, Fatimat Kudaeva ${ }^{2}$, Karamatilla Khayitboev $^{1}$, Zokhid Kusharov ${ }^{1}$, \\ Abrorjon Turgunov ${ }^{1}$, and Feruza Yusupova $^{1}$ \\ ${ }^{1}$ Tashkent State Agrarian University, 100140 Tashkent province, Uzbekistan \\ ${ }^{2}$ Kabardino-Balkarian State University named after H.M. Berbekov, 360004 Nalchik, Russia
}

\begin{abstract}
Nonlinear oscillations of single-mass system at force excitation of vibration connected with fixed base by weightless viscoelastic spring are considered. To take into account the rheological properties of the spring material, the Boltzmann-Volterr principle was used. Mathematical models of the problem in question are obtained, which are described by nonlinear integro-differential equations. A solution method based on the use of quadrature formulas has been developed and a computer program has been compiled on its basis, the results of which are reflected in the form of graphs. Influence of nonlinear and rheological properties of spring on amplitude and phase of mass oscillations is investigated.
\end{abstract}

\section{Introduction}

Vibrating machines, structures or their components are vibrating systems. One of the most important features of an oscillatory system is the number of degrees of freedom, i.e. the number of independent numerical parameters that uniquely determine the position of all points of the system in space at any fixed time moment $t[1,2]$.

To solve the problems of radiation protection in the design and operation of machines, equipment and structures, it is necessary to have dependences of the vibration parameters of their structures, excited by deterministic and random dynamic influences. In this case, the design model of structures can be taken as discrete or distributed (continuous), linear or nonlinear $[3,4]$.

The discrete model is characterized by the fact that all the masses of the structure are replaced by several lumped masses, the distributed and dissipative properties of the structure are also replaced by lumped elements of stiffness and inelastic resistance. The dynamics of discrete models is described by ordinary differential equations $[5,6]$.

Production activity in most branches of industrial production is provided by the work of various kinds of technological machines and vehicles. The operation of machines, equipment, mechanisms, apparatus and instruments in conditions of the need to ensure high productivity is often accompanied by significant dynamic loads, vibration processes and manifestations of shock interactions of machine elements. Ensuring the reliability and

\footnotetext{
* Corresponding author: nurnoralihaydar1964@,gmail.com
} 
safety of the operation of machines at all stages of their life cycle requires serious attention to the issues of compliance with certain restrictions on the parameters of the dynamic states of technical objects, the development of methods and tools for assessing the control and management of dynamic interaction processes $[6,7]$.

Many problems of statics and dynamics of mechanical systems admit relatively simple solutions based on linear differential equations. However, in a number of cases, it is necessary to take into account additional influences that force us to abandon the linear formulation of the problem and force us to investigate the so-called nonlinear systems. This problem is of great practical importance in many areas of technology $[8,9]$.

\section{Materials and methods}

Consider an oscillatory system with one degree of freedom in the form of a mass $(\mathrm{m})$ connected to a fixed base by a weightless nonlinear viscoelastic spring with a stiffness coefficient C (Fig. 1).

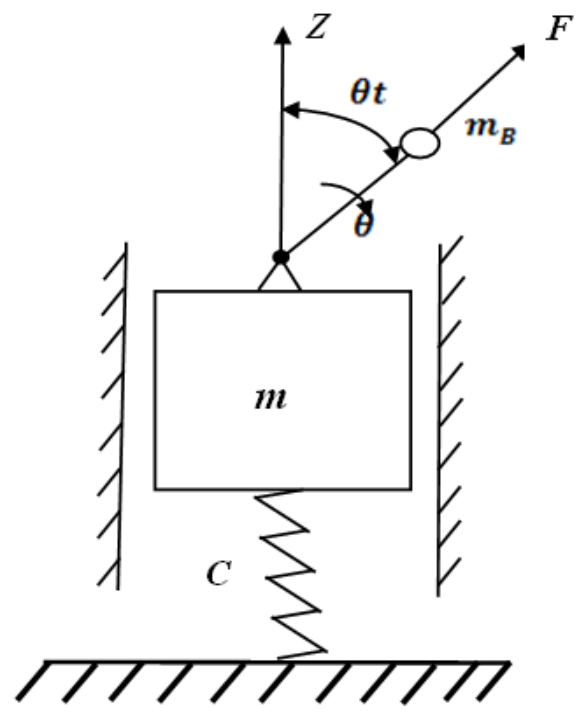

Fig. 1. Dynamic model of a single-mass system with power excitation of vibration.

The $m$ mass is acted upon by the inertial force of the unbalanced mass $m_{B}(1)$ :

$$
F(t)=m B e \theta 2 \cos \theta t
$$

where, $m_{B}$ - mass of the rotor of the machine, $\mathrm{kg} ; e$ - specific imbalance of the rotor, numerically equal to the distance of the center of mass of the rotor from the axis of its rotation, $\mathrm{m}$; and, $\theta$ - angular speed of rotation of the rotor, radian $/ \mathrm{sec}$.

The following forces act on the mass $m$ when it is displaced from the equilibrium position $Z(t)[5,6]$ :

$m Z$ - mass inertia force $m$;

$C R^{*}\left[Z(t)+\gamma Z^{3}(t)\right]$ - viscoelastic drag force;

$C\left[Z(t)+\gamma Z^{3}(t)\right]$ - elastic force.

where, $\gamma$ - nonlinearity coefficient; $R^{*}$ - integral operator with relaxation kernel $R(t)(2)$ : 
From the equilibrium of the system, taking into account the d'Alembert principle, we obtain a nonlinear integro-differential equation describing the fluctuations of mass (3):

$$
m Z(t)+C\left(1-R^{*}\right)[Z(t)+\gamma Z 3(t)]=m B e \theta 2 \cos \theta t
$$

Let's pretend that:

$$
Z(0)=0 ; Z^{\prime}(0)=0
$$

Introducing the following dimensionless quantities into (3):

$$
z / z x ; t / t x ; \gamma Z 2 x ; \theta t x t
$$

while retaining the previous notation and taking this:

$$
\omega 2=C t 2 x / m, p=m B t 2 x \theta 2 B / m Z x
$$

we get the following equation (4):

$$
Z(t)+\omega 2\left(1-R^{*}\right)[Z(t)+\gamma Z 3(t)]=p \cos \theta t
$$

Equation 3 is solved by methods based on the use of a quadrature formula [7-9]. Integrating $t$ twice according to equation (4), on the interval $[0 ; t]$ and taking into account the initial conditions (2), we have (5):

$$
Z(t)+\omega_{2} \int G(t-s) Z(s)[1+\gamma Z 2(s)] d s=p(1-\cos \theta t) / \theta 2
$$

where,

$$
G(t-s)=t-s-\int(t-s-\tau) R(\tau) d \tau ; R(t)=\varepsilon e-\beta t t \alpha-1
$$

Taking $t_{n}=n \Delta t,(i=0,1,2, \ldots)$ in the last expression and replacing the integrals with the quadrature formulas of the trapezoid, to determine the displacement of the load from the position $Z_{n}=Z\left(t_{n}\right)$, we have the following recurrent relations (6):

$$
Z n=p(1-\cos \theta t n) / \theta 2-\omega 2 \Sigma A i G(t n-t i)(Z i+\gamma Z 3 i)
$$

where,

$$
A 0=A n=\Delta t / 2 ; A j=\Delta t, j=1, n-1 ; G(t n-t i)=t n-t i-\int(t n-t i-\tau) R(\tau) d \tau
$$

\section{Results and discussion}

To carry out the computational calculation, a computer program has been developed, which the results obtained are reflected in the form of graphs. The calculation used the following initial data:

$$
\omega 2=5.6 ; \mathrm{p}=2.4 ; \theta=3.4 ; \beta=0.05 ; \varepsilon=0.05 ; \alpha=0.25
$$

The influence of the nonlinear viscoelastic properties of the spring on the displacement of the mass from the position of static equilibrium is investigated. Fig. 2 shows the effect of the nonlinearity parameter $\gamma$ on the mass vibration shape $m$. Here in the figure, $\gamma=0$ (solid 
line); $\gamma=2$ (dash line); and, $\gamma=3.5$ (dotted line). It can be seen from the graph that with an increase in the nonlinear property of the spring, the frequency increases, which comes at a phase shift to the left. Due to the nonlinearity of the spring, the amplitude of the mass oscillation increases [5-9].

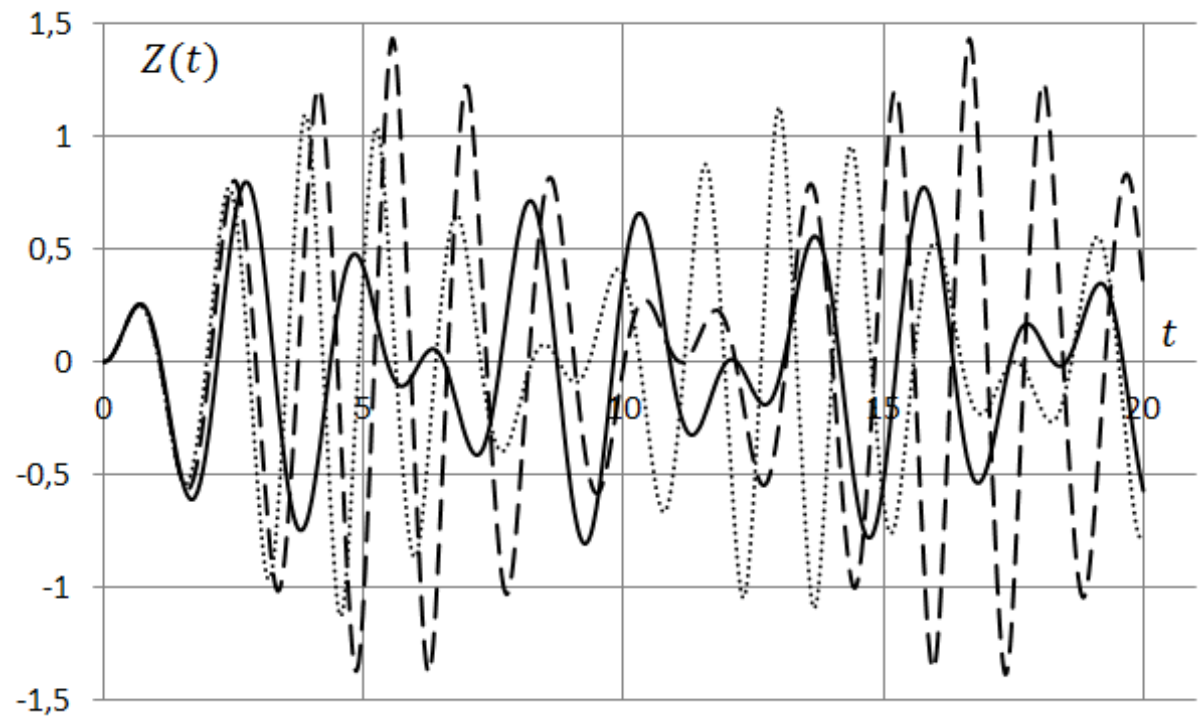

Fig. 2. Influence of the nonlinearity parameter $\gamma$ on the shape of the vibration by mass $m$.

Fig. 3 shows the influence of the parameter $\theta$ on the form vibration by mass $m$. Here, $\theta=3.4$ (solid line), $\theta=4$ (dash line), and $\theta=5$ (dotted line). Moving away $\theta$ from the natural frequency, the vibration amplitude decreases, and the vibration frequency increases. In this case, the natural frequencies of the mass are equal to 2.37 .

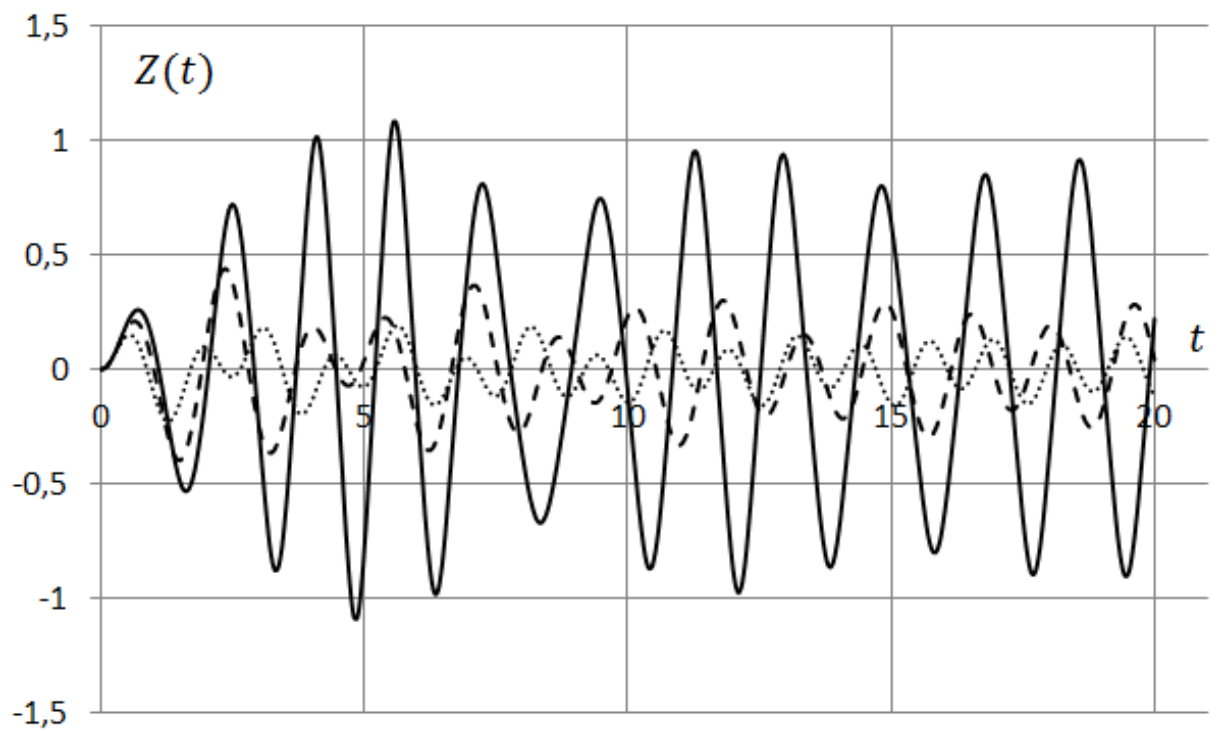

Fig. 3. Influence of the parameter $\theta$ on the shape of the vibration by the mass $m$. 
How do the rheological parameters of the spring affect the vibration modes? The change in rheological parameters $\varepsilon$ (Fig. 4) to the mode of vibration was investigated. The graphs are marked with: $\varepsilon=0$ (solid line), $\varepsilon=0.01$ (dash line), and $\varepsilon=0.05$ (dotted line). It can be seen from the graph that a small change in this parameter has a sensitive effect on the change in the oscillation frequency. The graph shows that the dependence of the parameter and the frequency of the oscillation are inversely proportional. This fact is explained by the fact that with an increase in the parameter, the spring material becomes more viscous.

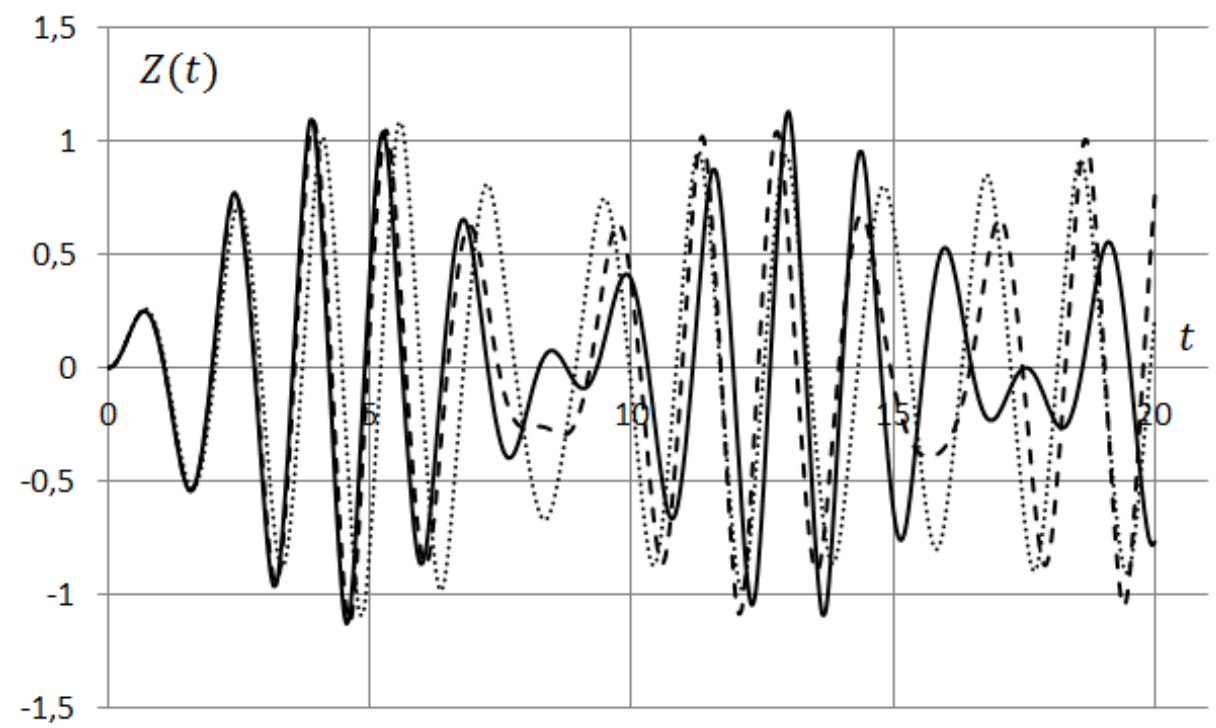

Fig. 4. Influence of the viscosity parameter $\varepsilon$ on the form of vibration by mass $m$.

\section{Conclusions}

Solving the problems of vehicle dynamics is associated with the need to carry out multiple calculations in the process of optimizing the parameters by devices for transforming motion. Therefore, in a number of cases, it is advisable to carry out preliminary calculations according to simplified design schemes to find out the approximate efficiency and parameters of devices for transforming motion. The use of schemes that allow obtaining a solution in a closed form or using algorithms is of great interest. The results obtained allow us to conclude that it is advisable to take into account the nonlinear viscoelastic properties of a spring during oscillatory processes of vehicles, both in ideal elastic and hereditarily deformable systems during transient processes.

\section{References}

1. N. V. Kutsubina, A. A. Sannikov, Theory of vibration protection and acoustic dynamics of machines, 167 (2014)

2. V. A. Shchepetilnikov, Mechanical engineering, 256 (1982)

3. G. S. Maslov, Mechanical engineering, 151 (1980)

4. Z. Abdullaev, M. Yusupov, S. Mirzaev, N. Noraliev, Z. Kusharov, IOP Conference Series: Materials Science and Engineering, 814(1), 014025 (2020) 
5. N. Noraliev, K. Sherenko, Prikladnaya Mekhanika, 27(12), 45-51 (1991)

6. N. Noraliev, B. Ishniyazov, B. Safarov, O. Saparov, E3S Web of Conferences, 258, 02014 (2021)

7. F. Kh. Kudaeva, N. Kh. Noraliev, Problems of Computational and Applied Mathematics, 4, 61-65 (2018)

8. B. Rakhmankulova, N. Noraliev, K. Djamalov, S. Ziyaeva, IOP Conference Series: Materials Science and Engineering, 814(1), 012047 (2020)

9. K. I. Sherenko, N. Noraliev, Strength problems, 9, 34-38 (1992) 